\title{
Enhancing Learning Through a Real-Life Assignment
}

\author{
Rolando Antonio Reyes Gonzalez ${ }^{1, a}$, Asle Fagerstrøm ${ }^{1 \& 2, b}$ and \\ Siri Fagernes ${ }^{1, c}$ \\ ${ }^{1}$ Westerdals Oslo School of Arts, Communication, and Technology (Faculty of Technology), \\ Christian Kroghs Gate 32, 0186 Oslo, Norway \\ ${ }^{2}$ Arcada University of Applied Science (Department of Business Management and Analytics), \\ Jan-Magnus Janssonin aukio 1, 00560 Helsinki, Finland \\ arolando.gonzalez@westerdals.no, basle.fagerstrom@westerdals.no, 'ciri.fagernes@westerdals.no
}

Keywords: Learning, real-life assessment, involvement, motivation.

\begin{abstract}
This study focuses on how a real-life assignment may enhance students' learning. Twenty-nine students completed an assignment to test a usability framework that small to medium enterprises (SMEs) in Nordic and Baltic countries can use to evaluate the usability of their website. The overall feedback from the students was that a real-life assignment like this was a positive experience with respect to enhanced motivation and understanding of typical issues in their educational field. This finding supports previous research, which shows that real-life assessments in higher education contribute to more satisfaction, which again can be the basis for future learning. Practical implications and suggestions for further research are given.
\end{abstract}

\section{Introduction}

Group projects, oral presentations, and peer assessments are methods used in higher education to enhance students' learning through the use of more realistic and meaningful tasks [1]. There is evidence that such non-conventional assessment methods have a positive impact on students learning, and students strongly support them. However, according to McDowell [1], innovative assessment is challenging within higher education in relation to understanding how it enhances learning. It is therefore important for the education sector to continue investigating how non-conventional assessment methods may affect students' learning. A better understanding of the motivating impact from non-conventional assessment methods may contribute to both research and practice in higher education.

Previous studies show that motivation is an important source for learning [2]. According to Keller [3], the educator can make use of several factors that create an environment that motivates the student to invest time and effort into achieving high performance. Factors that motivate students in their learning process are $[4,5]$ : (a) how they perceive the work they are given as a source of exploration, (b) that it is thematically relevant to what they study, and (c) that the work tests them in real-life tasks, that is, what they will be working with after finishing their education. When these three factors work together, they may have a very positive feedback loop as shown in Fig. 1 [3].

As can be seen in Fig. 1, effort affects the student's performance, which again affects the consequences (e.g., higher grades or better achievement). The level of effort, performance, and their consequences influence the degree of satisfaction the student may have; and this has an impact on the effort through a positive reinforcement of curiosity, motives, and expectancy. Therefore, assessments should be used not only as a way of evaluating the students, that is, as a measure of what they have achieved in knowledge and skills up to that point where the assessment starts, but also as an integrated part of their learning process. 
Personal Characteristics

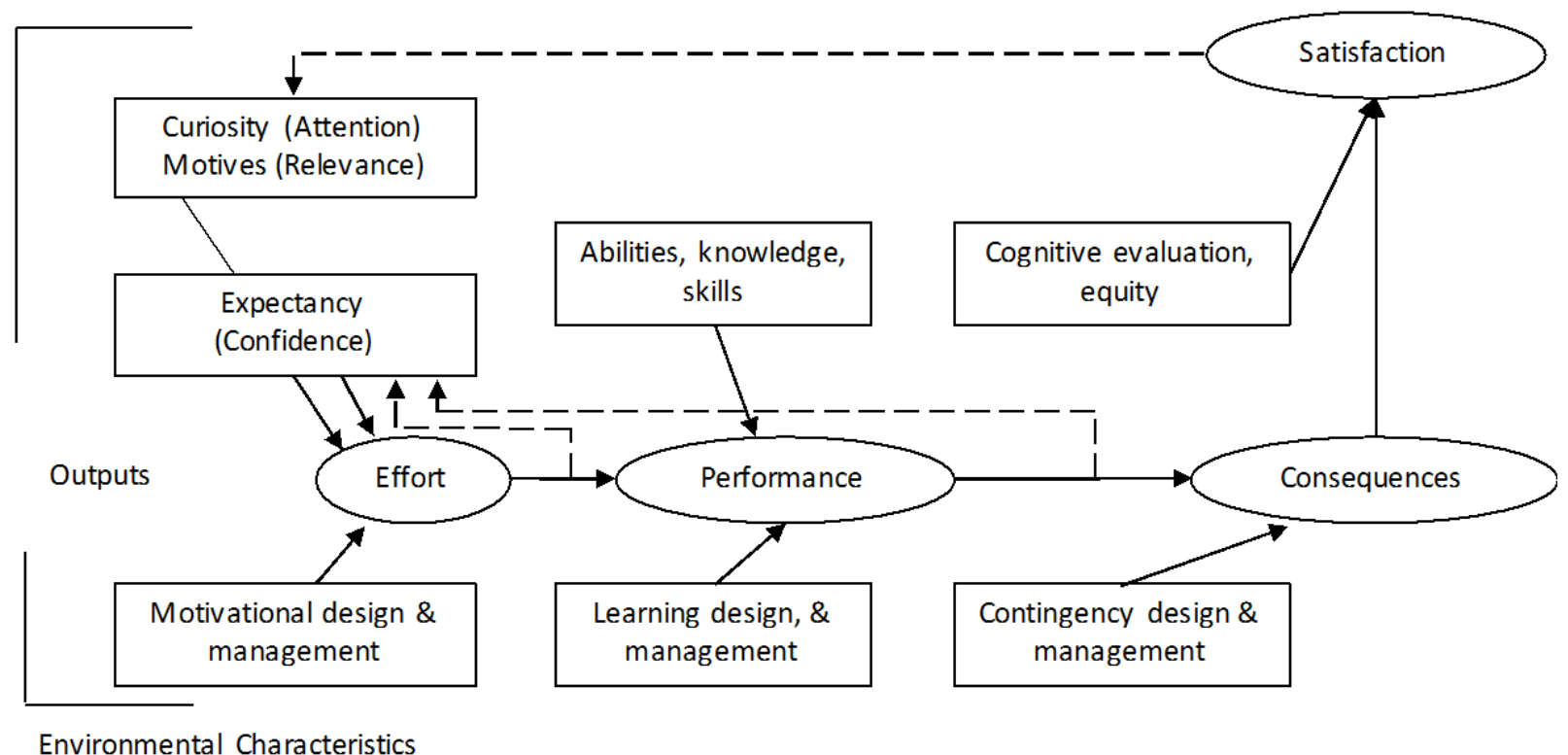

Fig. 1. Model of Motivation, Learning, and Performance by Keller [3]

Introducing real-life examples, exercises, and assessments in higher education contributes to a higher level of understanding and satisfaction, which again can be the basis for future learning [3, 4, 5]. It has also been demonstrated that real-life challenges increase students' motivation. In addition, real-life casework is a good way of introducing exercises that require the students to reflect critically upon their work, asking them about what they experienced while solving their tasks $[3,5]$. The overall aim of the present study is thus to contribute to the understanding of how a real-life assignment may enhance students' learning. This paper is structured as follows: First, the method design is presented. Next, the results are discussed and summarized. The paper concludes with a discussion of academic and managerial implications.

\section{Method}

A Nordic-Baltic Network for Internationalization of SMEs (NOBANET) was chosen as the basis for the study. NOBANET aims to create and widely disseminate new knowledge on successful internationalization of Nordic and Baltic small- and medium-sized enterprises (SMEs). This aim is achieved through close cooperation between higher education institutions and SMEs in all Nordic and Baltic countries. Faculty, students, and companies work closely together and across national borders, within educational courses and through real-life international assignments related to the industry.

An important issue when it comes to internationalization is how the company's website is presented. If the SME's website is not appropriately designed [6], there will most probably not be any internationalization. Usability guidelines for SME's in Nordic and Baltic countries are therefore needed, giving companies the opportunity to evaluate the usability of their own website. A usability framework was developed as a sub-project in NOBANET. This framework can be used by SMEs to check website usability related to design, user experience, internationalization, responsiveness, usability, stickiness, accessibility, and, search engine optimization.

As part of their assessment, third-year bachelor students at the Interactive Design program at Westerdals Oslo School of Arts, Communication and Technology were engaged to test the beta version of the usability framework. The assignment was presented as the first assignment in the course Effect Evaluation: Techniques and Tools, and as such, fits well since one of the learning outcomes was related to usability testing. The idea behind this real-life assignment was to increase students' motivation to learn. 
Thirty-one students were assigned to use the usability framework to evaluate one website from Finland and one website from Estonia. The criteria for the web company were that it should sell something to the consumer market and that the company website had its content translated into English. The assignment was presented on IT's Learning, the college learning management system. Given the assigned web companies, students were asked to evaluate the company websites based on the usability framework and to evaluate the framework itself. In addition, the students were asked to reflect upon how this real-life assignment worked in relation to their own learning process in the course (what did you learn, motivation, etc.). Student feedback in the latter task is the basis for our findings and discussion in the present study. The feedback from the students consisted of written responses to free-text questions and was collected and analyzed. Our method was textual analysis using an inductive approach. First, the responses were sorted according to whether they were positive or negative. As the large majority of the responses were positive, the few negative ones were studied in more detail, with the aim of getting a deeper understanding of why the students had negative experiences. The positive responses were then systematically read and sorted according to the themes that occurred multiple times. Experiences that seemed to be shared by several students are reported in this paper.

\section{Findings}

Twenty-nine out of thirty-one students, twenty males and nine females, completed the assignment. Most of the students were very positive in their feedback on how they experienced this assignment. Of all the students who responded, only two had negative comments. One student felt the motivation drop, but was quite vague in explaining why ("The pages were uninspiring to look at"). Another student did not see the point of using the guidelines; this person did not feel in charge of the evaluation process ("I felt like a test subject to evaluate a proposed guideline, and did not actually apply any of the material that I have learned in the course"). The negative responses indicated that some of the students did not see the relevance between evaluating websites using the provided guidelines, and the topics taught in the course.

The rest of the students seemed very happy with the overall experience. The opportunity to apply theory learned throughout their studies in a real-life scenario seemed to have been very motivating for many ("And this 'real life' assignment has given me motivation to make better websites in the future and try to never make these mistakes I have discovered. This gave me motivation for learning more about evaluating websites, and also other software's and apps"). Several students made comments that they had been motivated to search for more information on web usability and had found additional tools and guidelines to apply. Learning by doing, instead of merely reading or hearing about usability issues, was also mentioned as a positive experience.

The usability issues regarding international websites such as translation, cultural issues, developing good contact form and so forth, were also referred to as something with which most students did not have experience and that many found very meaningful: "What I learned by using the NOBANET usability guideline is the importance of internationalization, especially for creating great applications that have users from different countries, and why it is important to think of all the users while designing these kinds of applications. I am definitely more aware of what to keep in mind while designing applications and I will definitely check these guidelines for my next projects to come. " It was also mentioned that working on the assignment gave training in reflection and writing in English, which was perceived as a very useful.

Finally, several students commented on how they felt extra motivated by being able to discover weaknesses in actual websites and the idea that they could contribute to improving them in the end ("Developers can use these results to improve the usability of the websites in the future, which made me feel motivated and glad that my work could make a difference"). 


\section{Concluding Comments}

The aim of this study was to contribute to a better understanding of how a real-life assignment may enhance students' learning. Understanding students' learning processes may have an impact for both researchers and practitioners within higher education. Our findings show that students experience a better comprehension of typical issues in their future field of expertise and that they are more motivated to work on tasks that seem more "real." The negative feedback, even though it was limited, indicates that it must be very clear to the students how the real-life assignment is related to the defined learning outcomes of the course. If this connection is not obvious, the students seem less motivated to spend much time on the assignment.

We need to put emphasis on the negative feedback in this study. The feedback from the students was given as part of their individual assessment, and this may influence to what extent they are fully candid in their feedback. All positive feedback was justified; hence we have reasons to believe that it was real. However, students could have held back negative feedback, perhaps worried that this could affect their final grade in a negative way. To elicit more reliable results, the students should be able to give feedback on the process anonymously. Also, using more in-depth methods, such as more open-ended questionnaires and interviews, could provide a deeper understanding of the outcomes of this process.

Our findings are in accordance with how the factors in the model of Motivation, Learning, and Performance [3] are interconnected (see Fig. 1). The students' responses show that they reacted favorably to receiving an assignment including a relevant and real-life case. Overall, it seems to have had a positive impact on their motivation, which may indicate higher effort and improved performance through improved learning and reflection. Moreover, students' reflections show how the assignment inspired curiosity in the sense that they wanted to learn more, beyond what was required. Some of the students reflected on how they would use the experience they had attained in the future and how their work was meaningful in that it could be of value for the websites.

One of negative responses was about how the student did not find a connection between the assignment and being able to apply what was taught in the course, which is one of the factors affecting how motivated the student will be in working with an assignment [4]. However, the great majority of students seem to have found the assignment relevant to their studies and what they were to work with later and therefore gave feedback showing overall satisfaction working with the assignment.

\section{References}

[1] L. McDowell, The Impact of Innovative Assessment on Student Learning, Innovations in Education \& Training International. 32(4) (1995), 302-313. doi:10.1080/1355800950320402

[2] C. A. Catania, Learning (5th ed. ed.). (2013) Cornwall-on-Hudson, NY: Sloan Publishing.

[3] Keller, J. M. (2008). An integrative theory of motivation, volition, and performance. Technical Instruction, Cognition, and Learning, 6 (2), 79-104,

http://www.oldcitypublishing.com/FullText/TICLfulltext/TICL6.2fulltext/TICLv6n2p79-104K eller.pdf

[4] Williams-Pierce, Caro C. (2011): Five Key Ingredients for Improving Student Motivation, Mathematics \& Statistics at Scholars Archive at University at Albany, State University of New York, http://scholarsarchive.library.albany.edu/cgi/viewcontent.cgi?article=1000\&context=math_fac_ scholar

[5] Michael Theall, Jennifer Franklin (1999): What Have We Learned? A Synthesis and Some Guidelines for Effective Motivation in Higher Education, https://www.readcube.com/library/905f7218-ad07-4e31-977a-4a43d854cf22:abed1bdd-5e78-4 d95-a40c-abc46ff4134e

[6] P.-L. P. Rau \& S.-F. M. Liang. Internationalization and localization: evaluating and testing a Website for Asian users. Ergonomics, 46(1-3) (2003), 255-270. doi:10.1080/00140130303527 